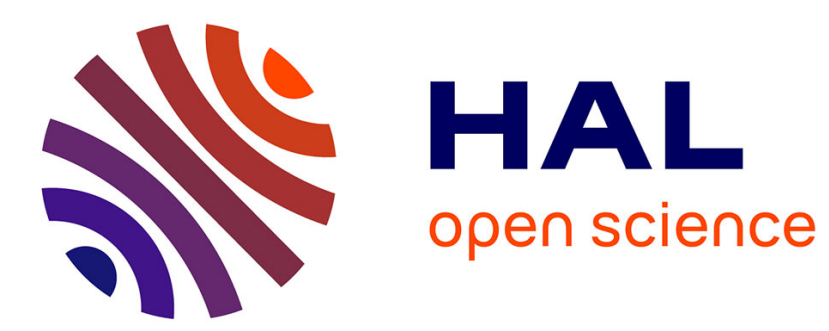

\title{
Devenir ouvriers en Amazonie
}

Doris Buu-Sao

\section{- To cite this version:}

Doris Buu-Sao. Devenir ouvriers en Amazonie. Terrains et Travaux: Revue de Sciences Sociales, 2019, 34 (1), pp.19-45. 10.3917/tt.034.0019 . hal-02385820

\section{HAL Id: hal-02385820 \\ https://hal-univ-rennes1.archives-ouvertes.fr/hal-02385820}

Submitted on 29 Nov 2019

HAL is a multi-disciplinary open access archive for the deposit and dissemination of scientific research documents, whether they are published or not. The documents may come from teaching and research institutions in France or abroad, or from public or private research centers.
L'archive ouverte pluridisciplinaire HAL, est destinée au dépôt et à la diffusion de documents scientifiques de niveau recherche, publiés ou non, émanant des établissements d'enseignement et de recherche français ou étrangers, des laboratoires publics ou privés. 


\title{
Devenir ouvriers en Amazonie \\ Entre travail industriel et vie de village
}

\author{
Doris BUU-SAO
}

\section{Résumé en français}

Cet article porte sur l'ouvriérisation de populations rurales confrontées à l'industrie pétrolière au Pérou, dans des villages amazoniens situés à proximité d'un site industriel actif depuis plus de quarante ans. Partant d'un dispositif d'accès à l'emploi dans les installations pétrolières - l'entreprise communale - qui consiste en la gestion, par les habitants, de leur propre main-d'œuvre, l'article interroge la portée des transformations causées par cette expérience particulière de l'emploi ouvrier, au moyen d'observations et d'entretiens ethnographiques. Soulignant les rapports inédits à l'environnement, au temps et à la discipline qui se tissent au sein de l'entreprise communale, il insiste dans le même temps sur la centralité des modes d'action villageois dans l'expérience du travail et de ses normes. L'analyse de l'appropriation de dispositifs, d'organisations et d'artefacts industriels permet alors de comprendre l'étendue du processus de socialisation, qui produit des effets transformateurs sur les travailleurs, mais aussi dans l'espace domestique et villageois.

Mots-clés : industrie pétrolière, ouvriérisation, hors-travail, Quechua, Amazonie péruvienne, communautés natives

\section{Résumé en anglais}

This article focuses on the workerisation process of rural populations facing oil industry in Peru, in Amazonian villages located near a forty-years old oil drilling site. Starting from the "communal enterprise", a device of jobs allocation that consists in inhabitants' management of their own workforce, the article scrutinises the range of transformations caused by such a peculiar experience of work, using ethnographic observations and interviews. While underlining new relations to environment, time and discipline within the communal enterprise, it insists on the influence of villagers' ordinary practices on their experience of work and norms. The analysis of the appropriation of industrial devices, organisations and artefacts thus allows to grasp the scope of the socialisation process, which produces transformative effects on workers, but also on the domestic and village spaces.

Keywords: oil industry, workerisation, non-work domain, Quechua, Peruvian Amazon, native communities 


\section{Ouvriers « à côté » \\ La socialisation des ouvriers-indigènes, entre travail et hors-travail}

L'Amérique latine est un terrain d'étude privilégié des mobilisations suscitées par l'industrie minière et pétrolière. De nombreux projets extractifs sont contestés par des groupes qui se définissent comme « indiens », « autochtones » ou « indigènes ${ }^{1}$ ». Mais si les moments de protestation favorisent la théâtralisation des antagonismes sociaux et des affiliations ethniques (Salazar-Soler 2016), l'observation des interactions routinières entre les sites industriels et les populations voisines révèle des configurations marquées par le jeu des transformations mutuelles (Ey et Sherval 2016), du fait notamment de dispositifs de «Responsabilité sociale des entreprises » (RSE) pensés pour pacifier les rapports avec les habitants en agissant sur leurs subjectivités (Billo 2015).

L'un de ces dispositifs, rarement étudié pour lui-même, fera l'objet de cet article : l'accès à l'emploi pétrolier. Il sera appréhendé à partir de l'ethnographie menée aux abords du plus ancien site pétrolier de l'Amazonie péruvienne ${ }^{2}$. À plus d'une semaine de voyage de la capitale et quelques kilomètres de la frontière avec l'Équateur, les habitants des « communautés natives ${ }^{3}$ » du fleuve Pastaza tendent à s'identifier aussi bien aux catégories de «indigène » ou « quechua »-le groupe ethnique majoritaire de la zone ${ }^{4}-$ que « travailleurs » et « ouvriers ». Car depuis 2000, la politique de RSE mise en œuvre par Pluspetrol, la compagnie qui exploite le gisement, favorise l'accès des habitants à l'emploi sur le site pétrolier. Il se fait majoritairement, depuis 2008, par le biais d'un dispositif conçu pour intégrer les populations rurales à l'économie de marché : des «entreprises communales », sortes de soustraitants créés et administrés par les habitants ${ }^{5}$.

Aujourd'hui, dans les communautés où il y a une entreprise communale, c'est devenu banal. On dit «c'est la compagnie », comme si de rien n'était. Mais avant on avait peur de la compagnie. Les femmes ne s'approchaient pas, c'était quelque chose de différent : des wirakucha, des métis... Non, elles ne s'en approchaient pas ${ }^{6}$ !

«Wirakucha» est un terme quechua qui, lors de la colonisation, désignait les Espagnols avec un mélange de déférence, de crainte et d'hostilité refoulée (Molinié 1987). Réapparu en Amazonie dans la relation avec les exploitants de caoutchouc ou de bois (Reeve 1988), ce terme désigne des étrangers blancs venus exploiter les ressources naturelles. Associé à celui de « métis ${ }^{7}$ », son usage indique que la perception des entreprises pétrolières est marquée du sceau de la subordination et de l'altérité - à plus forte raison du point de vue des femmes, face à l'immense majorité d'hommes employés par

\footnotetext{
${ }^{1}$ Ces termes (indio, indígena, autóctono) sont hérités de l'histoire de colonisation et de catégorisation des populations précolombiennes. Le stigmate a été réapproprié par les groupes ainsi catégorisés à partir des années 1990, à la faveur de l'institutionnalisation des droits des peuples autochtones à l'échelle internationale et d'un intérêt croissant de la part d'organisations de défense de l'environnement et/ou des droits de l'homme (Niezen 2003 ; Conklin 2006).

${ }^{2}$ L'analyse se fonde sur une enquête ethnographique de quinze mois menée entre 2012 et 2014 . La moitié du temps d'enquête a été consacrée à l'immersion dans des villages voisins du site pétrolier, tandis que l'autre consistait à suivre le processus de mobilisation contre la pollution industrielle. Ici, je mobilise essentiellement des observations et des entretiens avec des habitants de deux villages, dans lesquels j'ai effectué trois séjours de deux mois.

${ }^{3}$ Figure légale qui, depuis 1974, institue la reconnaissance de la propriété collective d'un ensemble de familles, au nom de leur appartenance à un groupe «ethnique», sur un territoire délimité. Cette propriété porte sur le sol mais pas sur le sous-sol ni ses ressources - qui appartiennent à la nation.

${ }^{4}$ Les Quechuas d'Amazonie sont issus de déplacements de population des Andes, à l'époque précoloniale, de regroupements dans des missions dominicaines qui ont utilisé le quechua comme langue franche (afin d'homogénéiser les pratiques linguistiques des groupes colonisés pour mieux les évangéliser), mais aussi de mouvements internes aux populations amazoniennes prises dans des processus de « fusion ethnique » (Whitten 1976).

5 J'ai analysé ailleurs la généalogie du dispositif de l'entreprise communale et son implantation locale (Buu-Sao 2018).

${ }^{6}$ Entretien avec Gabriel Cahuaza, habitant de Capahuari, âgé d'une cinquantaine d'années. Mai 2014, dans sa maison.

7 En Amérique latine, le terme désigne couramment les personnes qui ne sont ni «blanches » ni «indigènes » ou « noires ». Pour les habitants des communautés natives, il signifie souvent l'origine urbaine et plus favorisée.
} 
l'industrie pétrolière. Mais aujourd'hui, les entreprises seraient désormais «banales », intégrées à l'ordinaire villageois autrefois consacré à la chasse, à la pêche et à la culture sur brûlis.

Voilà qui évoque le « renversement de la vision du monde » observé par Pierre Bourdieu en Algérie, lors de la «conversion» des sociétés colonisées à l'économie de marché et à la raison calculatrice (Bourdieu 2003). Mais la « découverte du travail » par les habitants du Pastaza n'atteint pas le degré de violence qu'ont subie les paysans algériens, en proie notamment aux déplacements forcés. Elle s'éloigne également d'autres expériences amazoniennes de mise au travail coercitives et proches de l'esclavage, dans le cadre de l'extraction du caoutchouc à la fin du XIX ${ }^{\mathrm{e}}$ siècle (Barclay et Santos-Granero 2002) ou à l'époque coloniale, quand les indigènes servaient de main-d'œuvre captive à des missions assimilées à des « écoles industrielles » (Bolton 1917, p. 57). Aujourd'hui, les habitants du Pastaza sont ouvriers, gérants et propriétaires de l'entreprise communale, tout en maintenant (en partie) leurs activités productives rurales. À l'instar des paysans andins devenant mineurs (Salazar-Soler 2002), l'expérience de l'emploi industriel représente une rupture en termes de conditions de travail, mais aussi une continuité dans la mesure où elle s'ancre dans l'espace rural «traditionnel». Le processus de transformation n'agit pas sur des personnes vierges de toutes dispositions préalables ${ }^{8}:$ il s'articule à cette autre instance de socialisation qu'est la communauté native. L'entreprise communale s'inscrit dans l'espace habité où les villageois interagissent tantôt à titre de voisins ou parents, tantôt comme travailleurs insérés dans des relations hiérarchiques - en cela, elle se distingue des mines des Andes dans lesquelles se prolétarisent les paysans quechuas, au contact d'une hiérarchie venue de l'extérieur (ibid.). Les scènes sociales ${ }^{9}$ que sont l'espace du travail et celui de la communauté sont étroitement connectées : le statut d'ouvrier auquel les habitants s'identifient s'enracine dans l'espace villageois. Les ethnographies européennes soulignent combien les pratiques hors-travail, qu'elles relèvent de la «bricole », des loisirs, de la famille ou de l'espace domestique, sont centrales dans la constitution d'une culture ouvrière (Hoggart 1970 ; Schwartz 1990 ; Weber 2009). Si toute pratique économique peut être considérée comme « encastrée » dans des rapports sociaux (Polanyi, Arensberg et Pearson 1957 ; Barber 1995), dans le Haut Pastaza, les scènes professionnelle et résidentielle s'imbriquent d'une manière particulière autour de l'entreprise communale. La redéfinition du collectif à l'aune de son devenir «travailleur » prend appui sur des sociabilités extra-professionnelles qui perdurent au sein de l'entreprise.

Le présent texte interroge donc les processus de socialisation qui se jouent dans le cadre de l'entreprise communale, aux frontières du village et du monde industriel, en interrogeant à la fois la différenciation et l'interpénétration qui s'opèrent entre le travail et le hors-travail ${ }^{10}$. À rebours d'une lecture binaire et évolutionniste, il tient compte des jeux d'appropriation mutuelle qui caractérisent ce processus d'ouvriérisation ${ }^{11}$. Dans un premier temps, l'article reviendra sur l'émergence de pratiques et de normes spécifiques au monde industriel, auquel les entreprises communales donnent accès, et sur les transformations qui en découlent en termes d'organisation sociale. Mais ces transformations prennent appui sur la profonde intrication des scènes sociales que sont le travail en équipe et la vie de village. Dans un deuxième temps, l'analyse portera donc sur l'interpénétration de ces scènes sociales pour saisir la portée des transformations à l'œuvre.

8 Conformément aux approches qui tiennent compte de la pluralité des temporalités et des espaces de socialisation (Darmon 2006).

${ }^{9}$ Florence Weber parle de «scène sociale » pour désigner «le sous-ensemble des relations orientées par une pratique dans l'ensemble des relations que noue un individu » (Weber 2009, p. 157).

${ }^{10}$ On rejoint l'anthropologie économique qui considère la distinction entre le travail rémunéré et le travail domestique comme le fruit d'une construction sociale, et qu'il est nécessaire de tenir compte de l'inscription des activités de travail dans des pratiques sociales qui ne se réduisent pas à la sphère productive pour comprendre comment elles peuvent façonner les subjectivités des personnes qui les exécutent (Narotzky 2018).

${ }^{11}$ La notion d'appropriation mutuelle est d'ailleurs centrale pour P. Bourdieu quand il définit l'ouvriérisation comme « le processus par lequel les travailleurs s'approprient leur entreprise, et sont appropriés par elle, s'approprient leur instrument de travail et sont appropriés par lui » (Bourdieu 1984, p. 253). 


\section{« Entrer travailler » : devenir ouvrier sur le site pétrolier}

Dans le langage quotidien du Pastaza, l' «entrée » désigne une période de travail sur le site pétrolier, indiquant combien il s'agit de pénétrer dans un espace distinct. Alors que, depuis la découverte des gisements en 1971, les ouvriers urbains se rendaient dans les villages pour s'y divertir, à partir de 2000, les villageois ont la possibilité de travailler dans le monde pétrolier: d'abord engagés de manière rotative par des sous-traitants de Pluspetrol, puis plus systématiquement par l'intermédiaire d'entreprises communales qui ont leurs bureaux dans les communautés. Dans le village de Capahuari, où est créée la première entreprise communale en 2008, l'intégralité des 58 foyers qui le composent comptait au moins une «entrée » dans les derniers mois. Dans celui d'Andoas, où l'entreprise entre en activité un an plus tard, ils étaient $74 \%$ dans cette situation ${ }^{12}$. Ces «entrées » régulières sont l'occasion d'une socialisation professionnelle qui se décline selon les modalités formulées par Bernard Lahire : l'exécution répétée d'activités, l'agencement des situations et l'inculcation de normes spécifiques à l'instance socialisatrice (Lahire 2002, p. 421).

\section{Travailler au rythme de l'horloge}

Le domaine d'activité d'une entreprise communale s'étend à un ou plusieurs « fronts de travail ». L'expression, venue du génie civil, désigne une tâche dont est chargée une équipe composée d'une dizaine d'ouvriers, d'un infirmier et d'un contremaître. Lors des enquêtes de terrain, les entreprises communales pouvaient cumuler jusqu'à une dizaine de fronts de travail (soit plus de cent hommes employés) comme redescendre à un ou deux.

Les fronts de travail se déroulent dans deux types de lieux. Il peut s'agir d'ouvrages dans un des villages de la concession : réalisation d'un chemin bétonné, construction d'une place, etc. Ils sont financés par Pluspetrol, dans le cadre du programme de RSE, ou par l'entreprise communale qui doit réinvestir une partie des excédents au bénéfice du village. Une entreprise communale récemment créée se consacre d'abord à ce type d'œuvres, qui implique peu d'investissements. Mais à mesure qu'elle se développe, ses gérants obtiennent des fronts de travail sur le site pétrolier qui relèvent de la maintenance des installations : élagage, reforestation, entretien des routes, etc. Ces travaux, qui doivent être réalisés régulièrement sur l'ensemble du site compte tenu du climat tropical, en viennent à représenter la principale source d'emploi.

Ces activités peuvent sembler secondaires dans le cycle de production. Elles supposent cependant un rapport sensiblement différent à l'environnement. La photographie ci-dessous, prise à l'occasion d'une journée passée avec l'équipe "contrôle d'érosion » de l'entreprise de Capahuari, permet d'observer le travail réalisé sur une portion de ce front de travail. L'objectif était de protéger les infrastructures (la route, à droite, et l'oléoduc, à gauche) des altérations de l'environnement occasionnées par les averses quotidiennes. Ici, un glissement de terrain menaçait de provoquer l'affaissement de la piste et la rupture de l'oléoduc. Les ouvriers ont d'abord érigé ce qu'ils appellent une «banquette » à gauche de la route (de terre tassée recouverte d'une bâche de géomembrane ${ }^{13}$ ) pour réduire les risques d'affaissement. Ils ont ensuite défriché puis nivelé le bas-côté, créant de petites terrasses stabilisées à l'aide de planches débitées à la tronçonneuse dans des arbres abattus à proximité. Pour limiter la formation de boues avec l'écoulement des eaux de pluie, l'équipe a planté du gazon sur les terrasses. Enfin, ils ont surélevé les tubes sur des tréteaux métalliques pour réguler la tension à laquelle ils sont soumis.

\footnotetext{
${ }^{12}$ Il s'agit de données obtenues par la passation de questionnaires au porte-à-porte, dans 100 des 200 maisons qui constituaient le village d'Andoas en 2013, et dans la totalité des maisons de Capahuari en 2014.

${ }^{13}$ C'est un matériau très étanche, utilisé par exemple pour limiter la migration des particules polluantes lors de la fuite d'un oléoduc.
} 
Figure 1: Protéger l'infrastructure industrielle de l'environnement naturel

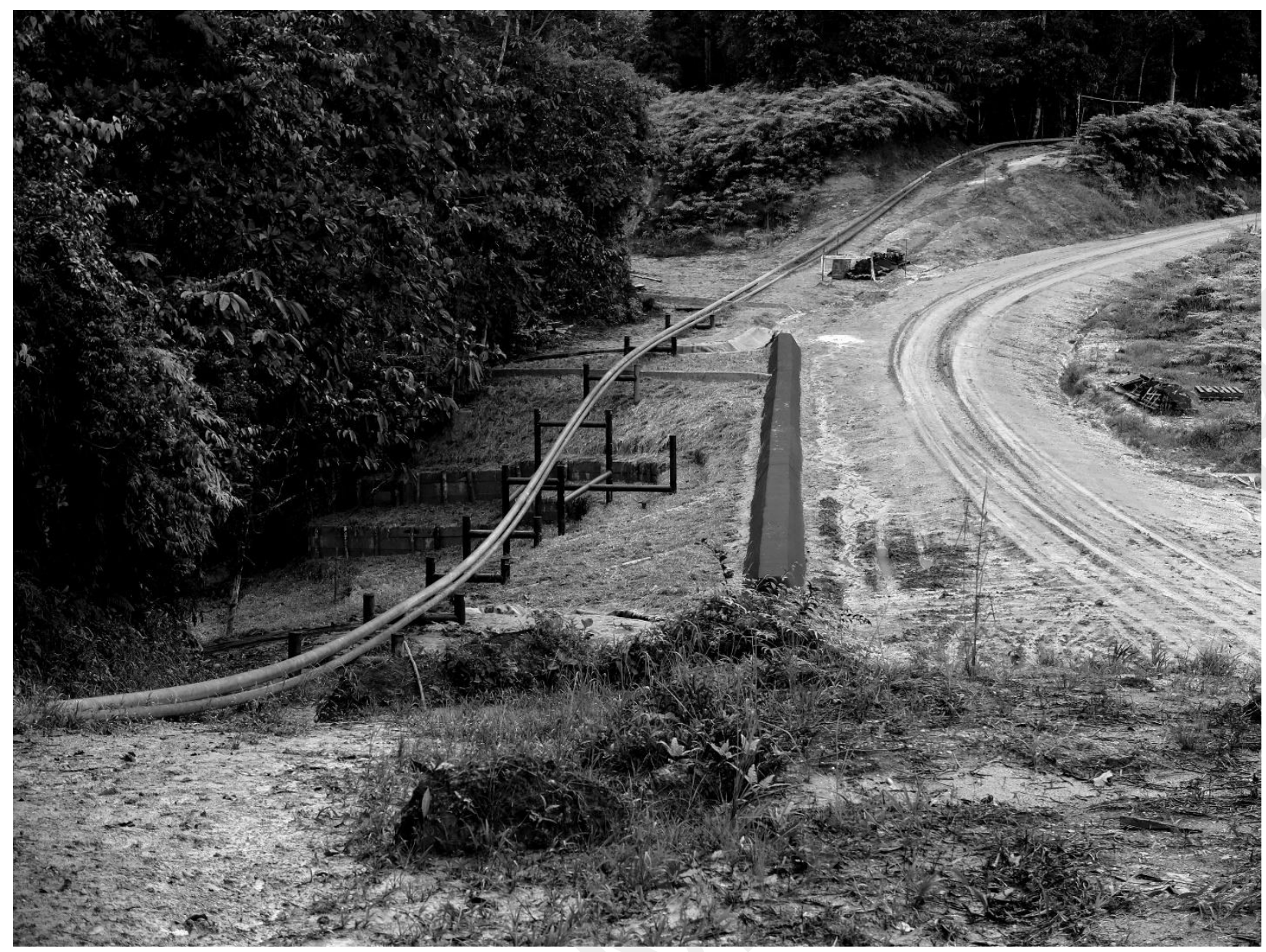

La réalisation de ces travaux n'est pas anodine: sans eux, de nombreux dysfonctionnements entraveraient la production. Surtout, cette activité n'a rien d'évident pour les habitants-ouvriers. Ces espaces sont parcourus pour les besoins de la chasse, de la cueillette ou de la pêche, par le passé mais aussi aujourd'hui, bien que dans une moindre mesure. Ce type d'usage n'implique pas une transformation majeure de l'environnement : un léger débroussaillage à la machette suffit à l'entretien d'un réseau de petits chemins imperceptibles aux yeux des citadins. Ce qui ne veut pas dire que la nature «sauvage » s'imposerait à ses habitants et déterminerait leurs pratiques. À l'encontre du déterminisme écologique, l'anthropologie de la nature initiée par Philippe Descola souligne l'existence de "modes de socialisation de la nature » spécifiques qui permettent d'établir une continuité entre l'espace domestique et l'espace naturel (Descola 2005). La maintenance des infrastructures suppose un autre type de socialisation de la nature. Le terrain argileux et le climat tropical sont peu propices à l'activité industrielle. En permanence, il faut abattre les arbres et élaguer régulièrement la végétation; lutter contre l'érosion des sols, elle-même favorisée par l'abattage des arbres ; contrôler la corrosion des tubes métalliques, soumis à l'humidité constante. Le rapport n'est plus de continuité mais de rupture : l'industrie doit l'emporter sur la nature.

Sans aller jusqu'à parler d'une « conversion » radicale à un schème de pensée opposé, il faut souligner cette dimension symbolique d'une activité dont le résultat ne se limite pas à la rémunération : le lieu de travail, comme tout autre espace de vie, est un espace de socialisation d'autant plus important qu'il est régulièrement arpenté par les habitants. La restitution d'une journée type de ces ouvriers permet de saisir comment ils prennent ainsi place dans l'espace pétrolier, par la nature des tâches qu'ils réalisent mais aussi par le rôle qu'ils sont amenés à endosser selon des temporalités spécifiques.

À 5h30, un murmure s'élève des maisons de Capahuari : une cinquantaine d'hommes se lève en même temps. Après une rapide toilette, ils enfilent leur uniforme (pantalon et chemise en jean), attrapent leur casque, leurs lunettes de protection et chaussent des bottes aux pointes métalliques fournies par 
l'entreprise. Puis ils se rendent à la maison où l'on « donne pension »: de manière rotative, des familles préparent trois repas quotidiens pour les travailleurs de l'entreprise communale. Le petit déjeuner est copieux : une soupe en entrée, un plat de viande ou de poisson accompagné de riz, manioc, banane et légumineuses ; des biscuits et une boisson rafraîchissante complètent le tout. La maîtresse de maison, sa famille ou des personnes employées pour l'occasion font le service. Les hommes se mettent en route vers 6h30. Pour rejoindre les « fronts de travail », il faut d'abord atteindre le réseau routier. Le solide repas n'est pas de trop pour endurer la petite heure de marche rapide à travers la forêt, sur un sol accidenté et boueux. Arrivés à la route, les hommes s'abritent sous une bâche pour attendre le pickup qui les emmène sur leurs fronts de travail respectif, où ils arrivent entre $8 \mathrm{~h}$ et $9 \mathrm{~h}$. Ils s'assoient sous une «tente » qui fait office de point de ralliement; quand tous les travailleurs sont présents, le contremaître ouvre le «speech » (charla) de sécurité. Une fois les tâches réparties, les ouvriers se mettent à l'ouvrage. L'infirmier distribue des boissons rafraîchissantes, le contremaître observe les opérations. À midi, le contremaître annonce la pause. Les travailleurs se rassemblent et sortent de leurs sac-à-dos les boîtes contenant leur repas. Ils se taquinent, évoquent des expériences partagées au village ou au travail. À $13 \mathrm{~h}$, le travail reprend. Vers $15 \mathrm{~h}$, le contremaître déclare la journée finie : les travailleurs doivent être rentrés à $17 \mathrm{~h}$. Arrivant par petits groupes au village, ils vont se baigner à la rivière. Certains s'éclipsent dans la maison d'un camarade pour partager une eau de vie ou de la bière de manioc appelée masato. Puis ils se retrouvent à la pension pour dîner, vers 19h. Certains s'y attardent pour regarder un film ou discuter. Mais vers $23 \mathrm{~h}$, le village est silencieux : les hommes sont partis se coucher, en prévision de la marche qui les attend le lendemain matin ${ }^{14}$.

La condition des travailleurs de l'entreprise communale rompt avec l'ordinaire des villages. Leur rythme de vie est plus proche de celui des autres travailleurs (de Pluspetrol et des entreprises soustraitantes) avec qui ils peuvent être confondus tant leurs uniformes sont similaires. L'organisation de la journée en horaires précis, les «entrées » de 28 jours et la prise en charge de l'alimentation sont des principes d'organisation du monde pétrolier. Au-delà de la gestion horaire de la journée qui remonte à l'organisation industrielle du travail (Thompson 2004), les « entrées » d'un mois suivies d'une semaine de repos sont propres au secteur extractif : elles n'ont de sens que parce qu'elles permettent aux ouvriers venus des villes de consacrer à l'ouvrage tout leur temps de présence sur la concession pour se réserver ensuite une semaine de repos auprès de leur famille ${ }^{15}$. De même, l'alimentation des ouvriers du lot, hébergés en baraquements, se justifie parce qu'ils ne bénéficient pas du travail domestique de leur épouse ${ }^{16}$. Cette organisation du travail est reprise par les entreprises communales, alors que les ouvriers ne se séparent pas de leur famille pendant leur « entrée ».

Travailler au rythme de l'horloge, malgré une chaleur amplifiée par l'uniforme, ou manger à heure fixe trois repas bien plus consistants que dans le quotidien des communautés : voilà qui n'a rien d'évident pour les habitants des villages, habitués à se nourrir de manière irrégulière, en fonction des aliments disponibles, à travailler « à la tâche » plus qu'à l'heure (ibid.), et à s'arrêter quand le soleil tape trop fort. «Il y en a beaucoup qui abandonnent, ceux qui ne sont pas habitués ; mais pour nous qui travaillons tout le temps, ça n'est plus rien », m'explique un soir Felipe, travailleur fréquent. Les propos de Felipe témoignent d'une certaine fierté : l'adaptation n'est pas facile. Certains comme lui sont à l'affût des « entrées » pour rompre la monotonie des journées passées entre la maison et la parcelle cultivée. Cette expérience répétée imprime dès lors une certaine discipline constitutive du rapport à soi.

${ }^{14}$ Journal de terrain, Capahuari, mai 2014.

${ }^{15}$ Le lot pétrolier comporte un aéroport à usage exclusif des compagnies, ce qui leur permet d'acheminer quotidiennement les salariés qui résident à Lima et Iquitos (autrement, le transport prendrait plusieurs jours, car le bassin du Pastaza - et même du Marañón, le fleuve dans lequel il se jette - est isolé de tout réseau routier).

${ }^{16}$ Dans les villages du Pastaza, les femmes préparent les repas, élèvent les enfants mais s'occupent aussi, de manière croissante, de l'entretien de la parcelle cultivée à mesure que les hommes consacrent une part plus importante de leur temps à l'emploi ouvrier. Voilà qui rejoint la tendance observée, dans les espaces miniers andins, à la féminisation des activités de subsistance rurales parallèlement à la masculinisation du travail salarié (Grieco 2016) - la plupart des hommes employés par l'entreprise communale continuent toutefois de pratiquer l'horticulture, la chasse ou la pêche en dehors des « entrées ». 


\section{Édifier un ordre hiérarchisé et sécuritaire}

Lors des sessions de travaux collectifs aux champs appelées minga, le « maître de la minga » se limite à indiquer l'ouvrage à réaliser aux amis et parents qu'il a convoqués. Quand l'un d'entre eux organise plus tard sa propre minga, celui qu'il a aidé aura l'obligation tacite d'accepter son invitation. Le rapport d'autorité est ainsi limité par le principe de réciprocité, qui détache le pouvoir d'injonction de la personne et le limite au strict minimum ${ }^{17}$. À l'inverse, l'organisation du travail au sein de l'entreprise communale repose sur une hiérarchie officielle qui détermine les prérogatives, les modes de rémunération ${ }^{18}$ et les lieux de travail de chacun. Le conseil d'administration, élu par l'assemblée générale, désigne les membres de la gérance. Ceux-ci se chargent d'engager les employés permanents et d'administrer la rotation des postes de contremaître, d'infirmier, d'ouvrier formé à l'exécution de tâches spécifiques et d'《 aide» (ouvrier non qualifié). En haut de cette hiérarchie se trouvent les gérants, des salariés à plein temps désignés par l'assemblée générale des sociétaires. En contact quotidien avec les travailleurs mais aussi avec leur «client», Pluspetrol, ils doivent faire preuve d'une « capacité à diriger », un savoir-faire qui ne va pas de soi dans ce contexte. La discipline concerne tout particulièrement les normes de sécurité, dont les gérants regrettent souvent qu'elles sont difficilement respectées « il faut convaincre les gens que ça, c'est une entreprise, que tu dois mettre tes gants - parce qu'avant ils ne mettaient pas de gants, que tu dois mettre tes lunettes, ton casque, ton uniforme, tes bottes; et ils doivent comprendre le pourquoi ${ }^{19}$ ». Les «moniteurs de sécurité »sont des employés permanents responsables de la mise en œuvre de ces normes imposées par Pluspetrol. L'un d'entre eux témoigne de l'importance de sa mission :

Un travailleur qui n'a pas d'accident, c'est un travailleur qualifié.

\section{C'est-à-dire qu'un travailleur qui a un accident, c'est un travailleur peu qualifié ?}

Peu qualifié. Et ça c'est ma spécialité, former les gens. [...] Tout ce qui concerne la sécurité. Prendre soin de soi au travail. Une entreprise n'a pas d'accident, c'est une entreprise qui ne manque pas d'emploi. [...] Pluspetrol te qualifie comme une entreprise qui travaille dans la sécurité, dont le personnel travaille consciencieusement. Et quand tu présentes ta candidature à un appel d'offre, tu gagnes ! Ils te donnent la priorité, parce que tu es une entreprise qui travaille de manière sûre, ton personnel connaît son travail et fait attention ${ }^{20}$.

Pour obtenir des contrats, il faut produire des «travailleurs qualifiés », autrement dit qui appliquent les consignes de sécurité. Les «moniteurs de sécurité » mettent en œuvre les conditions nécessaires au respect des normes : consignes générales lors de réunions hebdomadaires, gestion des stocks d'uniformes, d'équipements de sécurité personnelle (communément appelés «EPP») et des objets de signalisation, etc. Sur les sites de travail, les contremaîtres se chargent ensuite de rappeler les normes et de vérifier leur application lors de speechs quotidiens. Le personnel encadrant doit savoir faire respecter ces normes, ainsi que l'explique un ancien contremaître : "parfois, il y a des râleurs qui ne veulent pas respecter. Alors toi, comme contremaître, tu dois te lever, être une personne forte, leur dire en face "ça n'est pas bien" : les corriger $»^{21}$. Mais la sanction vient en dernier recours. Avoir suivi le «cours basique de sécurité » et effectué un examen de santé auprès du service de médecine du travail de Pluspetrol sont des conditions indispensables pour obtenir une «entrée». La

${ }^{17}$ L'évitement de la différenciation hiérarchique dans l'organisation des tâches productives est une modalité fréquente, dans les sociétés amazoniennes, pour empêcher l'émergence d'individus autoritaires et ainsi préserver l'"égalitarisme" souvent cher à leurs membres (Killick 2007).

18 À titre indicatif, d'après le gérant de l'entreprise de Capahuari, le personnel permanent et qualifié perçoit un salaire mensuel de $650 €$, les contremaîtres sont payés environ $500 €$, les ouvriers non qualifiés $450 €$, les lavandières $215 €-$ le service de la «pension » est pour sa part rémunéré à hauteur de $400 €$ par mois.

${ }^{19}$ Entretien avec Kevin Vasquez, gérant de l'entreprise communale d'Andoas, âgé d'une trentaine d'années et originaire du village. Juin 2013, dans sa maison.

${ }^{20}$ Entretien avec Virgilio Zúñiga, moniteur de sécurité de l'entreprise d'Andoas, âgé d'une quarantaine d'années et originaire du village. Juin 2013, dans sa maison.

${ }^{21}$ Entretien avec Mauricio Dahua, président de l'entreprise de Capahuari, âgé d'une trentaine d'années, originaire du village. Juin 2014, dans sa maison. 
signalétique dont sont responsables les moniteurs de sécurité, présente sur les fronts de travail mais également au cœur des villages, est une autre forme d'injonction au respect des normes.

\section{Figure 2: signalétiques sécuritaires}

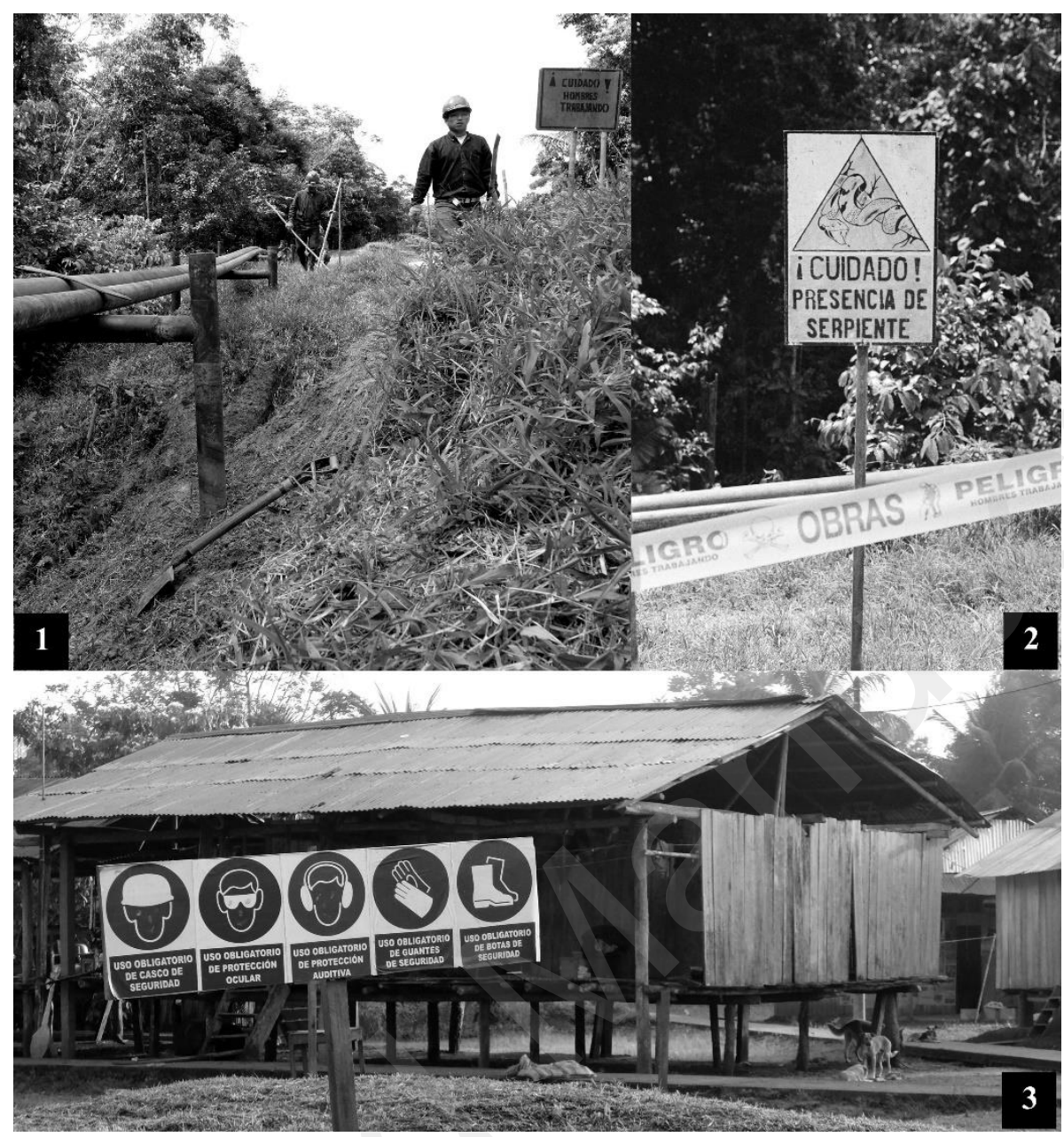

Les ouvriers concourent à la mise en scène d'un espace normé. Ainsi de la photographie ci-dessus (figure 2, vignette 1) où l'on voit des ouvriers affairés à l'installation d'une barrière pour délimiter de l'aire de travail, formée par des branches qui, plantées dans le sol, font office de piquets permettant de soutenir un ruban de sécurité (« danger - travaux »). Ils dressent des panneaux à intervalles réguliers pour avertir du chantier («Attention! Des hommes travaillent») et de ses dangers («présence de serpents »- vignette 2). Ces «éléments scéniques » constituent un «décor» dans lequel les ouvriers contribuent à l'édification d'une «façade » (Goffman 1973) par laquelle l'impératif de sécurité est mis en avant, sur la scène de travail mais aussi au cœur du village, comme l'indique l'installation au centre de Capahuari d'un panneau rappelant l'usage obligatoire de l'équipement personnel de sécurité (vignette 3).

Le speech donné par le contremaître de l'équipe « contrôle d'érosion » indique combien le processus de normalisation est ambivalent. Sous la tente dressée en bordure de la route, le contremaitre Walter est assis derrière une petite table faisant office de bureau, les autres en demi-cercle devant lui, sur des tronçons de bois. Il ouvre le speech après quelques minutes d'attente à échanger des plaisanteries en espagnol et en quechua au sujet des personnes absentes et des manières de contourner sa sanction, alors que la feuille de présence passe de mains en mains.

«Bon, on va parler du thème qui est là, annonce Walter en agitant dans sa main un formulaire. Avant tout, bonjour à tous. Le thème du speech est : sigles de sécurité. Des sigles de sécurité, il y en a cinq. Qui peut m'en donner un?»

Personne ne répond, le silence dure quelques secondes. 
«Bon. "STOP”, qui sait ce que ça signifie ? "STOP”, ça veut dire quoi ? Quelqu'un... ?», demande Walter. Quelques travailleurs murmurent "STOP” d'un ton évasif, les autres restent silencieux.

«Allez, qu'est-ce que vous dites? S-T-O-P...

- $\mathrm{S}$ pour sécurité..., avance un travailleur.

- $S$ pour sécurité, $T$ pour travail... suggère un autre.

- Euh, sécurité au travail... commence un troisième.

— Ce machin est écrit juste ici !, s'exclame un quatrième en pointant du doigt le sigle "STOP" cousu sur le bras droit de son vêtement de travail, provoquant un éclat de rire général.

- Et personne ne sait..., reprend Walter, d'un ton vaguement découragé.

— Mais si je sais, mais il y a tellement de sigles... », répond le troisième ouvrier, rieur.

Walter lit rapidement les consignes inscrites sur le document qu'il a sous les yeux. Après une dizaine de minutes, quand il a fini d'exposer les normes et les tâches à réaliser dans la journée, un travailleur intervient :

«Est-ce qu'il y a du masato ${ }^{22}$ pour...?

— Où est le masato ? demande Walter.

- Moi j'en ai amené, répond un travailleur.

— Quelqu'un d'autre en a amené ? relance Walter.

— Juste moi. Il y en a pour trois casques »

Nous partons vers la zone d'intervention ; je me charge de diluer la pâte de manioc fermenté dans un seau d'eau et d'imiter la manière qu'ont les femmes de distribuer la boisson. Un casque de sécurité fait office de récipient et les travailleurs le vident d'un trait, l'un après l'autre, jusqu'à ce que le seau soit vide. Le contremaître prend soin de le dissimuler derrière un talus, m'expliquant qu' « on ne sait jamais, des ingénieurs [de Pluspetrol] pourraient venir nous inspecter ». Quelques jours plus tard, Leoncio, le moniteur de sécurité de Capahuari, me confirme que la consommation de masato est interdite sur les fronts de travail, tout en étant conscient qu'elle y a cours.

À l'image de l'alcool à l'usine, la consommation de masato relève d'une transgression qui, ravivant la camaraderie et l'allégresse, réaffirme l'existence du collectif ouvrier face à l'ordre usinier (Pialoux 1992). Mais ici, le contremaître participe pleinement de la transgression. Selon l'analyse de la "gouvernementalité bureaucratique » que propose Béatrice Hibou, les normes ne s'appliquent pas mécaniquement aux sujets mais favorisent au contraire l'émergence d'informalités, autant de marges de manœuvre qui permettent à la norme bureaucratique d'advenir (Hibou 2012). Ici, le formalisme du speech va de pair avec le contournement de certaines normes par ceux-là mêmes qui sont censés les faire appliquer. La «force du formalisme », dans cette perspective, repose sur l'expression « de formules et de rituels souvent vidés de sens » (Hibou 2011, p. 63), et sur leur hybridation avec un quotidien spécifique aux populations visées. «Entrer dans l'espace pétrolier », c'est certes se plier à une discipline et s'engager dans des activités qui rompent avec les pratiques antérieures et insèrent les travailleurs dans un ordre distinct. Mais ceux-ci ne cessent jamais complètement d'être des amis, voisins ou parents.

\section{Par-delà « vie » et « travail », des principes de socialisation hybrides}

La différenciation formelle entre «vie » et «travail»n'empêche pas une étroite imbrication entre l'espace de la communauté et les fronts de travail. Comme en témoigne l'influence des pratiques coutumières sur le contournement des normes en vigueur sur le « front de travail », des manières de faire villageoises sont importées dans les pratiques de travail, et inversement ${ }^{23}$.

\footnotetext{
${ }^{22}$ Bière préparée artisanalement par les femmes qui font fermenter du manioc avec leur salive. En fonction du temps de fermentation, le masato peut atteindre un degré d'alcoolisation relativement important.

${ }^{23}$ On retrouve cet entremêlement des pratiques et espaces sociaux dans le cas de la prolétarisation des mineurs andins par C. Salazar-Soler (2002), mais aussi sur des terrains très distants tels que les usines en milieu rural français (Renahy, 2015).
} 


\section{La communauté dans l'entreprise}

On m'a souvent dit que les Quechuas du Pastaza étaient des personnes joyeuses. Sans aller jusqu'à attribuer l'exclusivité de ce caractère festif à l' " ethnie » quechua, les interactions quotidiennes sont bien souvent égayées par la malice et le rire. Surnoms moqueurs, exclamations joyeuses, histoires drôles répétées inlassablement autour du masato : s'amuser est un critère d'appréciation important tandis que, à l'inverse, le même verbe exprime l'ennui, l'inconfort comme la douleur ${ }^{24}$. L'appréciation d'une activité en fonction de l'ennui ou de la joie vaut aussi pour le travail.

Dans les scènes restituées plus haut, l'échange de plaisanteries est récurrent ; surtout, il est continu, à la différence d'autres situations de travail ${ }^{25}$. Depuis la marche matinale jusqu'à la pause déjeuner en passant par la réalisation des activités du jour, les conversations se poursuivent et les plaisanteries ne s'interrompent pas. Ainsi du temps d'attente qui précède un speech : entre deux éclats de rire, les travailleurs échangent des surnoms moqueurs en quechua comme «Felipe le menteur» pour celui qui a la réputation de tout exagérer, ou «Raul l'abîmé » pour un travailleur dont le goût pour l'eau de vie est connu de tous. Devant ces scènes d'allégresse, Felipe me dit avec fierté que «dans mon groupe, nous sommes tous joyeux ; personne n'est triste ${ }^{26} \gg$. La joie, modalité de présentation de soi au travail, est à la fois une motivation et un principe d'organisation au sein de l'entreprise communale : les responsables des « ressources humaines » tiennent aussi compte des affinités entre travailleurs pour composer les équipes.

Telle personne a telle spécialité, alors on la met là. [...] Par exemple, pour la «taille », ils sont tous de Capahuari. Parce qu'ils travaillaient là-dedans avant, ils avaient des notions, alors ils sont allés là-bas. [...] Andoas Viejo ${ }^{27}$ va surtout dans le groupe d'érosion parce que pour ça on utilise beaucoup la pelle. Et eux, ils sont très bons avec la pelle. [...]. Et c'est comme ça qu'ils se répartissent, parce qu'ils aiment ça, travailler en groupe. Avec leur cousin, de chaque communauté. Pour qu'ils puissent discuter, dialoguer... se sentir bien dans leur travail. Pour qu'ils ne soient pas mécontents ${ }^{28}$.

La composition des équipes articule un principe rationnel, la mise à profit des compétences préalables des travailleurs, et un principe d'affinité : faire en sorte que les membres d'une équipe «se sentent bien dans leur travail ». Ici, le moniteur personnalise les villages, les citant comme s'il s'agissait de personnes dotées d'attributs personnels : au principe de la constitution des communautés se trouve la parenté, dont les réseaux sont préservés dans la constitution des équipes.

$\mathrm{Au}$ caractère presque ludique de ce quotidien ouvrier, imprégné de sociabilités villageoises extraindustrielles, il faut ajouter que le rythme de travail contraste avec les fortes contraintes horaires imposées par la discipline usinière. En tenant compte des temps de déplacement, des pauses obligatoires pendant les pluies et de l'heure de repas, le temps de travail effectif se limite parfois à deux heures ; le reste de la journée est consacré à la marche et aux échanges entre les membres de l'équipe. La rupture instituée entre «la vie » et « le travail », si elle constitue une réelle ambition pour les gérants de l'entreprise, doit donc être nuancée ${ }^{29}$. D'autant que les tâches réalisées sur les « fronts de travail » ne sont finalement pas très éloignées de celles réalisées dans le quotidien villageois : scier des arbres et en faire des planches, sarcler à la machette, débroussailler, etc. Un sens différent est

\footnotetext{
${ }^{24}$ L'usage du verbe aburrirse en espagnol (littéralement : s'ennuyer) m'a initialement surprise, quand les villageois s'en servaient pour se plaindre de la chaleur, du froid ou de la douleur. En quechua aussi, les verbes amichina et killarina associent tous deux l'idée d'ennui et celle de douleur ou d'inconfort physique.

${ }^{25} \mathrm{La}$ « pause casse-croûte » marque par exemple un moment d' «antitravail » et de « dispersion de l'équipe » qui permet aux ouvriers de rétablir leur identité dont ils sont en partie dépossédés durant le travail à la chaîne (Hatzfeld 2002).

${ }^{26}$ Journal de terrain, Capahuari, mai 2014.

${ }^{27} \mathrm{C}$ 'est un village qui ne dispose pas d'entreprise communale. On verra par la suite que le recrutement ne se limite pas toujours au seul village où les entreprises sont implantées.

${ }^{28}$ Entretien avec Leoncio Dahua, moniteur de l'entreprise communale de Capahuari, âgé d'une trentaine d'années, originaire du village. Mai 2014, dans sa maison.

${ }^{29}$ Pour une analyse similaire, ancrée dans une ethnographie qui amène à nuancer les conclusions de l'article de Thompson sur le temps et la discipline en usine, voir Parry (2002).
} 
attribué à ces activités, avec la prégnance de consignes de sécurité qui incitent à envisager en permanence la possibilité d'un accident. Mais quand la machette reste le principal instrument de travail, qu'elle est maniée collectivement, dans une ambiance joyeuse que nourrit la consommation de masato, alors le travail évoque plus la minga que l'usine disciplinée. La similitude des pratiques mais aussi de l'environnement physique des «fronts de travail » avec celui des autres activités productives limite dès lors l'effet de rupture observé par Carmen Salazar-Soler (2002) lors du passage des paysans andins des parcelles cultivées aux sous-sols de la mine. D'autant que les modalités d'accès à l'emploi s'inscrivent, elles aussi, dans les sociabilités villageoises.

Les questionnaires confirment que tous les sociétaires le souhaitant travaillent ponctuellement comme ouvriers. Mais certains ont plus souvent l'occasion de se voir proposer une « entrée ». Par ailleurs, à mesure que s'accroît le nombre de «fronts de travail », les gérants sont amenés à ouvrir l'accès à l'emploi au-delà du réservoir habituel de travailleurs, parmi les non-sociétaires du village comme d'autres communautés du lot. L'obtention de ces « entrées » pour les non-sociétaires dépend souvent de la qualité de leurs relations avec les gestionnaires des entreprises communales. Ainsi de Ricardo Chino, ancien $a p u^{30}$ d'une communauté de l'aval qui s'installe à Andoas en 2013 pour y solliciter un emploi. Francisco, en tant qu'apu d'Andoas, est alors chargé de recruter les personnes extérieures à la communauté. À cette époque, leurs relations sont plutôt amicales et il obtient un poste sans difficulté. Mais un an plus tard, ils se sont distanciés. Il est donc parti s'installer à Capahuari, chez son neveu par alliance, Alejandro. C'est le frère de l'apu, dont un fils et un frère sont moniteurs de sécurité de l'entreprise.

Pour travailler, il faut que tu ailles quémander à l'apu, « allez apu, apu, inscris-moi encore une fois »; tu dois lui offrir sa bière ! [...]

Oui? Ah, si tu ne fais pas ça, il ne te...

Il ne va surtout pas te recommander. C'est pour ça que là-bas, je n'ai pas travaillé un an complet. [...] Et c'est là que mon neveu Alejandro vient me voir et il me dit « viens mon oncle, ici [à Capahuari] il y a du boulot, on manque de monde ». [...] J'y suis allé, et l'apu [de Capahuari] était en train de boire un coup par là-bas, et il m'a dit « cousin, il y a du boulot, il y en a beaucoup ! - Mais tu vas m'en donner, mon oncle ? » je lui dis. « $\mathrm{Oui}^{31}$ !»

L'obtention de ce bien rare qu'est l'« entrée »s'inscrit dans des logiques qui articulent une économie des faveurs à des réseaux de parenté. L'existence de sociabilités qui ne s'embarrassent pas des frontières instituées entre «le travail » et «la vie ${ }^{32} » n$ 'est bien sûr pas spécifique à ce seul terrain. Même dans les régions industrialisées de longue date, les « rapports de travail ne sont finalement qu'une des modalités qui relient des personnes prises, d'avance ou parallèlement, dans un milieu structuré et hiérarchisé d'interconnaissance où se font et défont des réputations, des obligations, des échanges »(Weber 1992, p. 3).

\section{L'entreprise dans la communauté}

Les entreprises communales impriment leur marque sur le quotidien des communautés, qui peut être interprétée en termes de «culture matérielle». Les artefacts, produits physiques d'un travail de transformation humaine, de la nature, jouent en retour un rôle central dans les processus de socialisation : " le sujet ne peut pas être isolé du monde matériel à l'intérieur duquel et par lequel il est constitué » (Miller 1997, p. 86). Ainsi, la fréquentation régulière des installations pétrolières favorise l'appropriation d'artefacts industriels qui contribuent à redéfinir l'espace villageois et

\footnotetext{
${ }^{30}$ Terme quechua utilisé pour désigner les chefs de village (officiellement, « présidents de communauté native »).

${ }^{31}$ Entretien avec Ricardo Chino, dans la maison (vide) de son neveu Alejandro, juin 2014, Capahuari.

${ }^{32}$ Dans la perspective d'E. Thompson (2004), la discipline industrielle repose sur l'édification d'une frontière entre la sphère de «la vie » et celle du «travail », institués comme deux espaces séparés avec des activités, des temporalités et des normes distinctes.
} 
l'habitat. L'usage de ces objets s'inscrit d'abord dans une tendance à la privatisation de l'espace. Dans les villages de l'aval, les maisons ne comportent qu'une ou deux cloisons pour aérer l'intérieur. Il n'est pas de frontière étanche entre la maison, le petit jardin et les maisons voisines, souvent habitées par la famille proche. Dans les villages voisins du site pétrolier, les habitants tendent à cloisonner leur maison et leurs jardins. Des bidons aplanis et cloués les uns aux autres pour édifier les murs, complétant les plaques de tôle ondulée distribuées par les « relations communautaires » de Pluspetrol (figure 3 ci-après, vignette 1). Les habitants récupèrent aussi la géomembrane utilisée sur le bloc pétrolier quand manquent les bidons et la tôle ondulée : tendue contre la charpente des maisons, elle achève de clore l'espace domestique.

Figure 3 : Artefacts industriels dans les villages

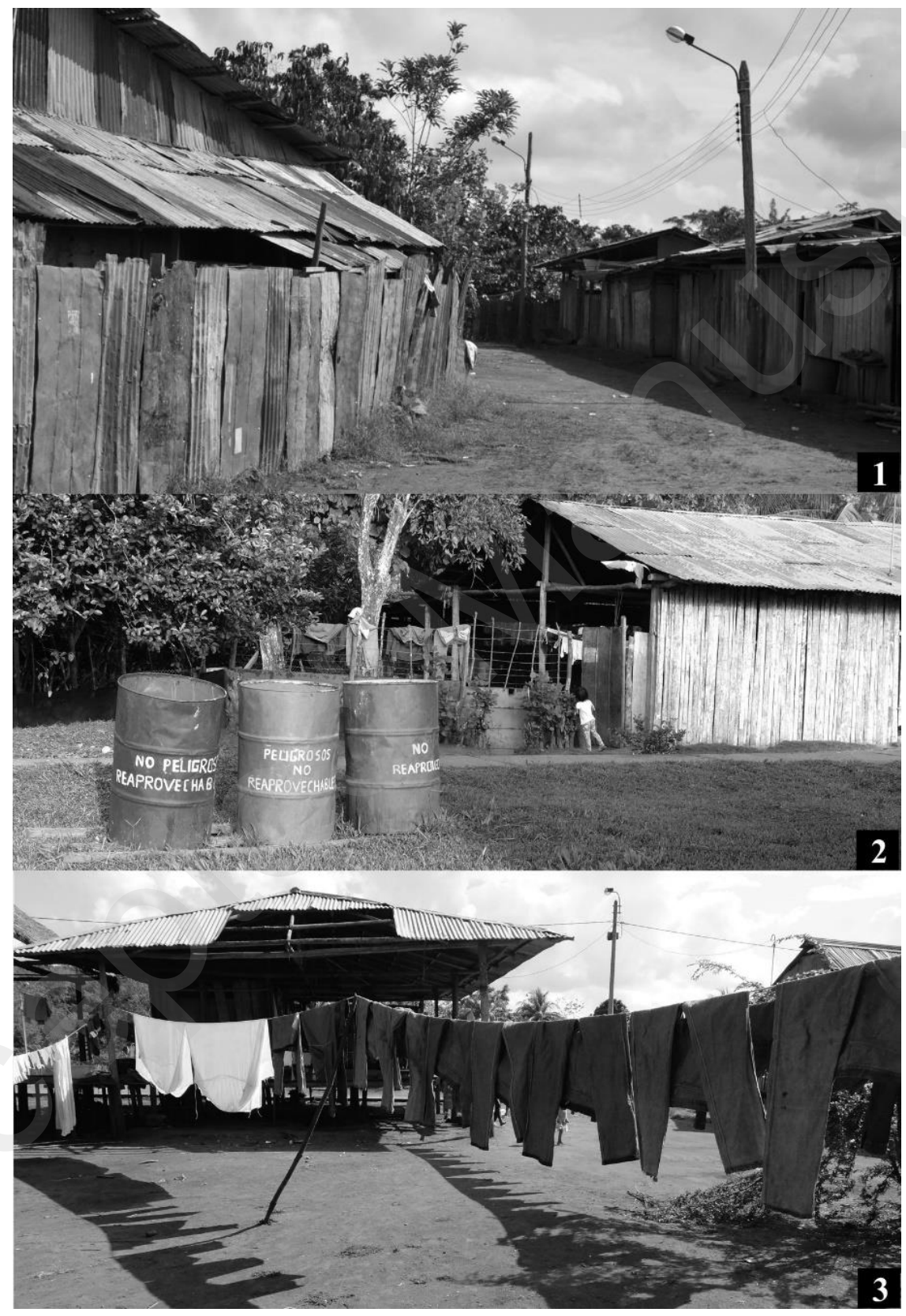

S'observe également une tendance à la normalisation des espaces communs. « Nous allons arranger ce chemin, pour qu'il soit droit au lieu de zigzaguer », m'annonce un jour Mauricio, le président de l'entreprise de Capahuari, désignant le chemin de terre qui débouche sur le sentier emprunté pour accéder à la route : il souhaite «faire venir les machines pour urbaniser le village ${ }^{33}$ ». Peu à peu, les

${ }^{33}$ Journal de terrain, Capahuari, mai 2014. 
communautés natives adoptent un plan en damier dont le cœur est composé d'une « place d'armes », à l'image des villes péruviennes. L'idéal de « civilité » et de «bonnes mœurs » que convoie le tracé rectiligne de la ville coloniale (Durston 1994) est formulé à nouveaux frais, rencontrant les imaginaires locaux du progrès et du développement ${ }^{34}$. Les gérants sont les premiers à soutenir ces projets : l' « urbanisation » représente des «fronts de travail» supplémentaires. Les moniteurs de sécurité prennent eux aussi en charge la mise en ordre des lieux publics : ils récupèrent des bidons vide pour trier les détritus, s'inspirant du système de classification industrielle (« dangereux, non recyclable », « recyclable »- figure 3 , vignette 2 ).

Les uniformes qui sèchent en permanence le long des fils tendus entre les maisons (figure 3, vignette 3), utilisés pour se rendre au front de travail, servent aussi aller chasser en forêt ou cultiver la parcelle, où ils protègent efficacement des piqûres d'insectes. L'intérieur domestique est habité par des objets venus du monde pétrolier : literie et ustensiles de cuisine marqués des noms de Pluspetrol et de ses sous-traitants participent de la structuration de l'habitat suivant des fonctions précises. Toute une série d'artefacts industriels peuplent ainsi le quotidien des familles, accompagnant leurs pratiques de l'habitat comme de la production alimentaire.

La transposition d'objets dans l'organisation spatiale des villages et de l'habitat témoigne de l'introduction de manières de faire, de principes d'action spécifiques aux entreprises communales. Les sessions de «travail communal», forme collective de réalisation de tâches dans les lieux communs évoquées plus haut, permettent d'observer cette transposition en actes.

Un matin d'avril à Capahuari, à 7h, l'« agent municipal », l'autorité villageoise responsable de l'ordre communal, rappelle au haut-parleur une décision prise lors de la dernière assemblée : mis à part les «travailleurs », tous les hommes et femmes du village suspendront aujourd'hui leurs activités pour participer au travail communal. Les collégiens aussi participent, car le travail consiste à préparer pour eux la construction d'une «auberge d'étudiants». En effet, le collège de Capahuari accueille des élèves venus d'autres villages; alors qu'ils sont de plus en plus nombreux, les villageois ont décidé qu'il faudrait les héberger dans un lieu spécifique.

A $8 \mathrm{~h} 30$, les participants se réunissent au centre du village pour recevoir les directives. L'apu et le président de l'entreprise communale expliquent les étapes du travail à réaliser : il faut d'abord transporter les planches, débitées par les travailleurs de l'entreprise communale, jusqu'au terrain de construction de l'auberge. Elles sont rassemblées devant le bureau de l'entreprise communale, une maison de deux étages dressée au centre du village. Il faut ensuite défricher le terrain, à la machette et à la tronçonneuse. Les femmes sont chargées de préparer le repas, avec les produits remis par chaque famille. Ricardo Chino est responsable de la tronçonneuse : ouvrier-tronçonneur pour le compte de l'entreprise communale, il a suivi une formation à Pluspetrol pour réaliser cette tâche. Leoncio Dahua, moniteur de sécurité de l'entreprise, a été nommé « contremaître »; il participe au défrichage tout en surveillant son déroulement, vêtu de son pantalon de travail et muni d'un casque de chantier ${ }^{35}$.

Lors de ces sessions de travail communal, une série de manières de faire évoque les entreprises communales. D'abord, la discipline : les autorités cherchent à mettre en œuvre une participation systématique et un respect des horaires. Faire respecter les directives est une préoccupation de premier plan pour les autorités. Ainsi du bilan que Leoncio fait de son mandat d'agent municipal, qui a pris fin trois ans plus tôt : «j'ai mis en place un règlement. [...] Le travail commence à $8 \mathrm{~h}$ pile. Après 10 minutes de tolérance, on ne t'accepte plus et tu paies l'amende. Si tu ne viens pas deux fois de suite,

\footnotetext{
${ }^{34}$ Karolien Van Teijlingen (2016) rend compte de projets urbanistiques similaires dans des communautés indigènes (shuar) d'Amazonie équatorienne, face aux mégaprojets miniers et aux discours du développement qui les entourent.

35 Journal de terrain, avril 2014, Capahuari.
} 
on te met au cachot $[\text { calabozo }]^{36}$ de la communauté. Et la communauté s'est élevée, ça l'a améliorée, ce respect de l'ordre ${ }^{37}$.» L'ordre disciplinaire est ainsi au fondement de l' «élévation» de la communauté, et non plus seulement de l'entreprise communale.

Figure 4 : Contremaître et ouvrier-tronçonneur au travail pour la communauté

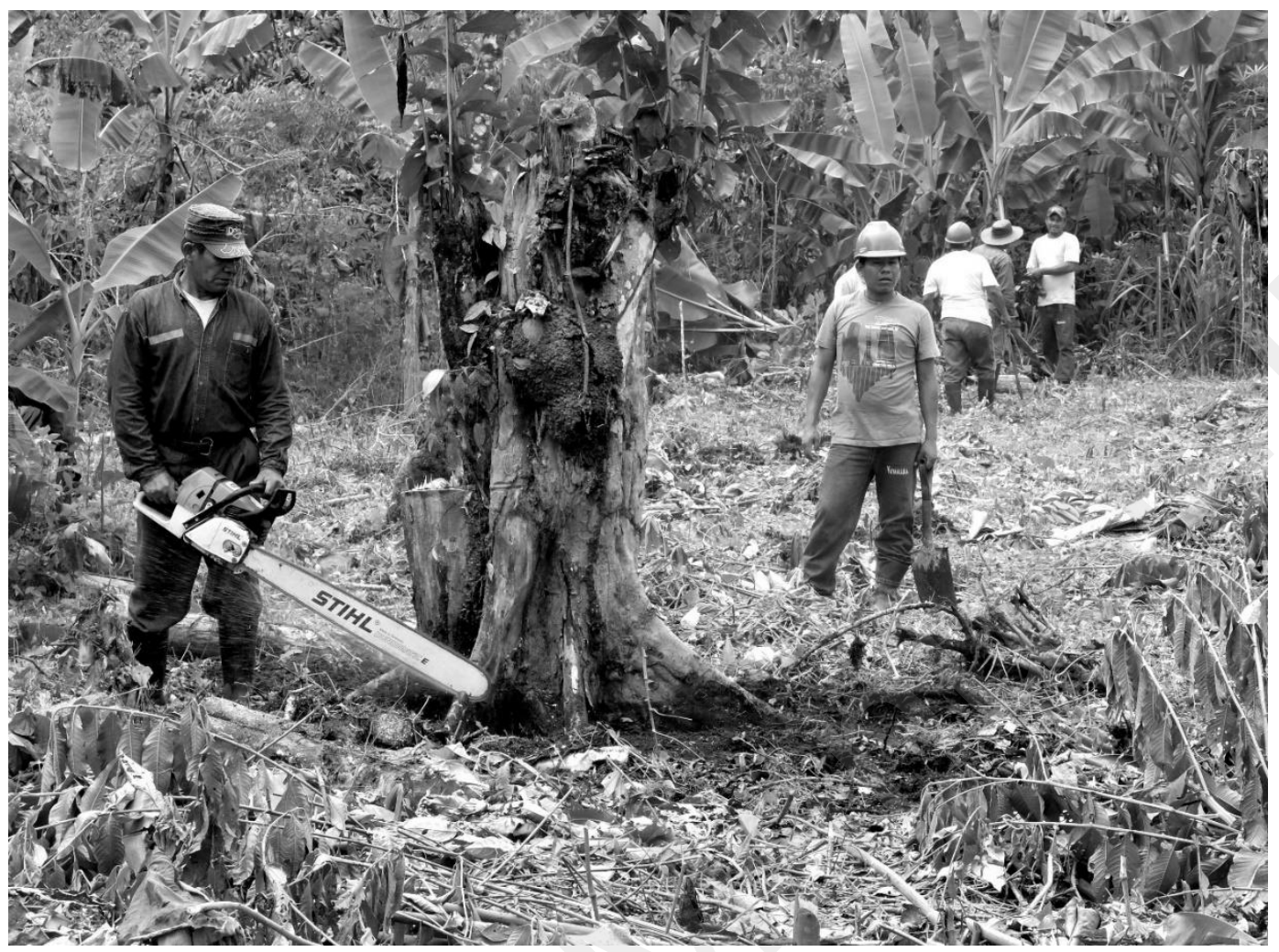

Est aussi repris le principe de division du travail et de hiérarchisation des rôles : les compétences certifiées par Pluspetrol sont réinvesties dans l'organisation du travail communal ; les femmes endossent la fonction de «cuisinières » pour assurer la «pension» des habitants, délaissant le défrichage qui est traditionnellement une activité mixte ${ }^{38}$. L'espace lui-même est pensé selon des fonctions spécifiques. Le travail communal consiste à édifier une auberge dédiée aux élèves : il s'agit de les concentrer en un seul lieu où s'appliqueront des règles spécifiques, à distance des lieux de diversion du village. Les villageois manifestent ainsi leur souci de contrôler l'activité des collégiens pour qu'ils se concentrent sur leurs études ${ }^{39}$.

Enfin, l'entreprise occupe une place centrale dans l'organisation du travail communal. Ce sont à la fois les autorités du village et les responsables de l'entreprise communale qui dirigent les opérations. Ce rôle transparaît aussi dans le soutien logistique à la réalisation des travaux. Les planches de bois sont coûteuses en temps de travail : il faut se rendre en forêt, abattre les arbres, débiter les planches une par une, à la tronçonneuse, puis les transporter. Que l'entreprise fournisse ces planches témoigne d'une réutilisation des ressources matérielles (outils de travail) et humaines (ouvriers) au profit de la communauté. L'entreprise communale n'est pas uniquement une voie d'accès au travail dans le complexe pétrolier : elle doit être une source de croissance pour l'ensemble du village.

\footnotetext{
${ }^{36}$ Le calabozo dont les communautés natives sont souvent équipées est un petit cachot de bois dans lequel il n'est parfois possible de tenir que debout. L'envoi au calabozo pour une durée variable constitue une sanction fréquente en cas de manquement aux règlements de la communauté.

${ }^{37}$ Entretien cité avec Leoncio Dahua.

${ }^{38}$ Lors de la session de travail communal dont il est question ici, seule une femme participait au défrichage ; c'était une des plus anciennes du village.

${ }^{39}$ On pourrait à nouveau établir un rapprochement avec l'analyse de Thompson, pour qui l'école joue au XIII ${ }^{\mathrm{e}}$ siècle le rôle de préparation des enfants à la discipline de l'horloge (Thompson 2004).
} 
Nous, en tant qu'entreprise, nous nous occupons du travail, mais c'est important aussi en tant qu'entreprise de veiller à la santé et à l'éducation. [...] Les enseignants que tu vois ici, ils sont payés par nous, par l'entreprise. [...] C'est important de soutenir l'éducation, non ? Et aussi, maintenant nous payons un infirmier [...]. En cas d'urgence, il aide avec les médicaments, avec les premiers soins [...]. C'est important de soutenir la communauté, de la soutenir socialement ${ }^{40}$.

Parce que l'entreprise communale dispose de ressources, elle a la responsabilité d'en redistribuer une partie à l'ensemble du village. La redistribution passe par la réalisation d'ouvrages pour la communauté, par des formes de soutien logistique ponctuel mais fréquent, voire même parfois par le versement de petites sommes aux personnes âgées. Sont aussi créées des « entrées » réservées à des catégories de personnes dans le besoin. À Andoas, le gardiennage des locaux de l'entreprise communale est réservé aux « anciens », tandis que les mères célibataires ont la priorité pour se charger de la lessive des uniformes. À Capahuari, les hommes âgés peuvent s'employer à collecter les détritus qui jonchent le sol du village. La rationalité économique est loin d'être la seule à dicter les critères d'accès à l'emploi : interviennent aussi le sens de la responsabilité, la volonté d' « aider » les familles selon une évaluation de leurs besoins et de leur mérite. Alors qu'aucune des personnes âgées du village ne bénéficie de la retraite théorique récemment instaurée et que de nombreuses femmes doivent élever seules des enfants conçus avec des ouvriers qui ne sont pas restés, l'entreprise communale se substitue en partie aux fonctions sociales de l'État.

\section{Conclusion}

La socialisation au travail ouvrier en Amazonie péruvienne est finalement diffuse et nuancée. La condition de «travailleur » s'accompagne d'un rapport spécifique à l'environnement, au temps et à la discipline. Mais la mise en œuvre des normes de sécurité et leur détournement indiquent combien cette socialisation ne se réduit pas à une imposition unilatérale : l'ordre n'advient que parce qu'il est réapproprié et parfois transgressés par les ouvriers-indigènes. Dès lors, il s'agit moins d'une rupture radicale que d'une socialisation hybride, plus encore que dans les mines des Andes où le travail en sous-sol introduit une organisation radicalement différente des activités productives (Salazar-Soler 2002). L'ambivalence de cette socialisation au travail nourrie de sociabilités villageoises permet l'extension de ses effets au-delà du seul collectif des « travailleurs » : progressivement, les pratiques et artefacts issus du monde industriel sont transposés au village, exerçant leur influence sur la vie domestique et villageoise.

Ces transformations sont donc profondément collectives, soulevant alors la question des significations politiques du travail dans les villages amazoniens. Dans la continuité des travaux qui soulignent le rôle toujours central du travail, dans son articulation avec le hors-travail, dans la production de subjectivités politiques (Sainsaulieu et Surdez (dir.) 2012 ; Narotzky 2018), l'analyse menée ici met en lumière des formes d'identification collective, de soumission et d'insoumission nourries par l'emploi au sein des entreprises communales. Les habitants des communautés natives se définissent de manière croissante comme « travailleurs » voire « ouvriers »; l'entreprise communale devient un cadre structurant du quotidien de ses employés comme de l'organisation sociale du village. Ce qui ne veut pas dire que les habitants se fondent dans une condition ouvrière uniformisée : l'ouvriérisation hybride des habitants du Pastaza constitue un socle ambivalent pour affirmer une identité indigène particulière, à la lisière de l'industrie et des villages ${ }^{41}$. Alors qu'en introduction, cet article suspendait provisoirement l'étude des conflits sociaux pour s'intéresser au quotidien des interactions routinières entre les sites extractifs et les populations environnantes, il est peut-être temps

\footnotetext{
${ }^{40}$ Entretien avec Calixto Dahua, gérant de l'entreprise communale de Capahuari, âgé d'une trentaine d'années et originaire du village. Mai 2014, dans sa maison.

${ }^{41}$ En cela, la situation amazonienne détone avec l'analyse de Paulo Drinot (2011), qui appréhende la formation de l'État péruvien à l'aune de la construction et de l'incorporation d'une classe ouvrière conçue comme non-indigène, bien que majoritairement d'origine andine - construction qui a permis d'instituer une frontière symbolique durable entre la modernité industrielle, associée à la figure de l'ouvrier, et le primitivisme supposé des Indiens.
} 
de reposer la question des formes de protestation qui surgissent, malgré tout, dans des espaces où l'emploi ouvrier et l'intégration au cycle de production pétrolier tendent à devenir un horizon partagé.

\section{Références}

BARBER Bernard, 1995, «All economies are "embedded". The career of a concept, and beyond », Social Research, vol. 62, $\mathrm{n}^{\circ}$ 2, p. 387-413.

BARCLAY Frederica et SANTOS-GRANERO Fernando, 2002, La frontera domesticada. Historia económica y social de Loreto, 1850-2000, Lima, Fondo editorial PUCP, 546 p.

BILLO Emily, 2015, «Sovereignty and subterranean resources. An institutional ethnography of Repsol's corporate social responsibility programs in Ecuador », Geoforum, vol. 59, p. 268 277.

BOLTON Herbert, 1917, «The mission as a frontier institution in the Spanish-American colonies », The American Historical Review, vol. 23, $\mathrm{n}^{\circ}$ 1, p. 42-61.

BOURDIEU Pierre, 2003, «La fabrique de l'habitus économique », Actes de la recherche en sciences sociales, vol. $150, \mathrm{n}^{\circ} 1$, p. $79-90$.

BouRdiEU Pierre, 1984, Questions de sociologie, Paris, Minuit, 277 p.

BuU-SAO Doris, 2018, «Indigènes et entrepreneurs. Le capitalisme au village », Sociologie du travail, vol. 60, $\mathrm{n}^{\circ} 3$, https://journals.openedition.org/sdt/2705.

ConkLin Beth A., 2006, «Environmentalism, Global Community, and the New Indigenism » dans Max Kirsch (ed.), Inclusion and Exclusion in the Global Arena, London, Routledge, p. 61-176.

DARMON Muriel, 2006, La socialisation, Paris, Armand Colin, 127 p.

DESCOLA Philippe, 2005, Par-delà nature et culture, Paris, Gallimard, 640 p.

Drinot Paulo, 2011, The Allure of Labor. Workers, Race, and the Making of the Peruvian State, Durham, Duke University Press, 311 p.

DURSTON Alan, 1994, «Un régimen urbanístico en la américa hispana colonial. El trazado en damero durante los siglos XVI y XVII », Historia, vol. 28, p. 59-115.

Ey Melina et SHERVAL Meg, 2016, «Exploring the minescape. Engaging with the complexity of the extractive sector $»$, Area, vol. 48, n 2 , p. 176-182.

GofFMAN Erving, 1973, La mise en scène de la vie quotidienne, Paris, Minuit, vol. I. La présentation de soi, $372 \mathrm{p}$.

GRIECO Kyra, 2016, «Le "genre" du développement minier. Maternalisme et extractivisme, entre complémentarité et contestation », Cahiers des Amériques latines, $\mathrm{n}^{\circ}$ 82, p. 95-111.

HATZFELD Nicolas, 2002, « La pause casse-croûte », Terrain. Revue d'ethnologie de l'Europe, $\mathrm{n}^{\circ}$ 39, p. 33-48.

HiBou Béatrice, 2012, La bureaucratisation du monde à l'ère néolibérale, Paris, La Découverte, 223 p.

Hibou Béatrice, 2011, Anatomie politique de la domination, Paris, La Découverte, 298 p.

HOGGART Richard, 1970, La culture du pauvre. Étude sur le style de vie des classes populaires en Angleterre, Paris, Minuit, $424 \mathrm{p}$.

KILlick Evan, 2007, «Autonomy and leadership. Political formations among the Ashéninka of Peruvian Amazonia », Ethnos, vol. 72, nº 4, p. 461-482.

LAHIRE Bernard, 2002, Portraits sociologiques. Dispositions et variations individuelles, Paris, Nathan, $431 \mathrm{p}$.

Miller Daniel, 1997, Material Culture and Mass Consumption, Oxford et New York, Basil Blackwell, $252 \mathrm{p}$.

MOLINIÉ Antoinette, 1987, «El regreso del viracocha », Bulletin de l'Institut Français d'Études Andines, XVI, n ${ }^{\circ}$ 3-4, p. 71-83.

NAROTZKY Susana, 2018, « Rethinking the concept of labour », Journal of the Royal Anthropological Institute, vol. $24, \mathrm{n}^{\circ} 1$, p. 29-43.

NIEZEN Ronald, 2003, The Origins of Indigenism. Human Rights and the Politics of Identity, Berkeley, University of California Press, 304 p. 
PARRY Jonathan P., 2002, « Du bagne des champs aux riantes usines. Le travail dans une entreprise sidérurgique indienne », Terrain. Revue d'ethnologie de l'Europe, $\mathrm{n}^{\mathrm{o}} 39$, p. 121-140.

PiAlOuX Michel, 1992, «Alcool et politique dans l'atelier. Une usine de carrosserie dans la décennie $1980 »$, Genèses, vol. 7, nº 1, p. 94-128.

Polanyi Karl, ARENSBerg Conrad et PEARSON Harry (eds.), 1957, Trade and market in the early empires. Economies in history and theory, Glencoe, Illinois, Free Press, 382 p.

REEVE Mary Elizabeth, 1988, «Cauchu uras. Lowland quichua histories of the Amazon rubber boom »dans Jonathan David Hill (ed.), Rethinking History and Myth: Indigenous South American Perspectives on the Past, Urbana, University of Illinois Press, p. 19-34.

RENAHY Nicolas, 2015, «Le village dans l'usine. Trajectoires ouvrières et mises à distance de la hiérarchie », Sociétés contemporaines, vol. 99-100, n 3, p. 65-80.

SAINSAUliEU Ivan et SuRdeZ Muriel (eds.), 2012, Sens politiques du travail, Paris, Armand Colin, $320 \mathrm{p}$.

SALAZAR-SOLER Carmen, 2016, «La place de l'ethnicité dans les conflits miniers socioenvironnementaux dans les Andes du Pérou : XXème-XXIème siècles », IdeAs, $\mathrm{n}^{\circ} 8$.

SCHWARTZ Olivier, 1990, Le monde privé des ouvriers, Paris, Presses Universitaires France, 531 p.

THOMPSON Edward P., 2004, Temps, discipline du travail et capitalisme industriel, traduit par Isabelle Taudière, Paris, La fabrique, $104 \mathrm{p}$.

VAn TEIJLINGEN Karolien, 2016, "The "will to improve" at the mining frontier. Neo-extractivism, development and governmentality in the Ecuadorian Amazon », The Extractive Industries and Society, vol. 3, n ${ }^{\circ}$ 4, p. 902-911.

WEBER Florence, 2009, Le travail à-côté. Une ethnographie des perceptions, Paris, EHESS, 238 p.

WEBER Florence, 1992, «Lieux du travail », Genèses, vol. 7, nº 1, p. 2-4.

WhitTen Norman E., 1976, Sacha Runa. Ethnicity and Adaptation of Ecuadorian Jungle Quichua, Urbana, University of Illinois Press, 348 p. 